\title{
Efeitos de um programa cinesioterapêutico na autonomia funcional de idosas
}

Marine Fernanda Lima Gama, José Newton Lacet Vieira, Sarah Tarcisia Rebelo Ferreira de Carvalho

\section{UNIVERSIDADE CEUMA}




\title{
Efeitos de um programa cinesioterapêutico na autonomia funcional de idosas \\ Effects of a program physiotherapists in the autonomy of elderly
}

\author{
Marine Fernanda Lima Gama; José Newton Lacet Vieira²; Sarah Tarcisia \\ Rebelo Ferreira de Carvalho ${ }^{3}$
}

\section{Resumo}

Introdução: Autonomia funcional constitui elemento fundamental para independência de idosos, referentes às atividades físicas e mentais necessárias para manutenção de atividades de vida diária (AVD'S). Objetivo: Avaliar os efeitos de um programa cinesioterapêutico na autonomia funcional de idosas. Material e métodos: Estudo intervencionista, com 20 idosas, de $70,5 \pm 67$ anos de idade, boa cognição e equilíbrio, do Centro de Referência em Assistência Social, em São Luís-MA. Utilizou-se protocolo de avaliação da autonomia funcional do Grupo de Desenvolvimento Latino-Americano para a Maturidade (Protocolo GDLAM), antes e após a intervenção. A intervenção consistiu em programa cinesioterapêutico adaptado de Montenegro e Silva (2007), com exercícios que simulam AVD'S, de 60 minutos, uma vez por semana, durante oito semanas. Para análise dos dados, utilizou-se estatística descritiva. Resultados: Observou-se diminuição do tempo necessário para que as participantes realizassem todos os testes do protocolo, no entanto, estes índices não foram suficientes para alterar a classificação da autonomia funcional das idosas, que continuou sendo fraco. Em relação ao teste levantar da cadeira e locomover-se pela casa, as idosas evoluíram de regular para bom. Conclusão: os resultados sugerem que o programa cinesioterapêutico pode ter colaborado para que as idosas diminuíssem o tempo de realização dos testes do protocolo GDLAM, melhorando, assim, autonomia funcional delas. Porém, os escores não foram suficientes para alterar a classificação quanto aos padrões da GDLAM em todos os testes. Sugerem-se novas pesquisas de caráter longitudinal, com número maior de sessões do protocolo.

Palavras-chave: Envelhecimento; Autonomia Funcional; Cinesioterapia.

\section{Abstract}

Introduction: Functional autonomy is a fundamental element of

Fisioterapeuta, graduada pela Universidade Ceuma.

2

Professor Mestre do Curso de Fisioterapia da Universidade Ceuma.

Professora Doutora dos Cursos de Fisioterapia e Estética da Universidade Ceuma. 


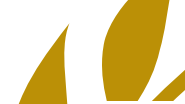

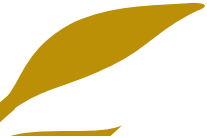

independence of the elderly, related to physical and mental activities required for maintenance activities of daily living (AVD's). Objective: To evaluate the effects of a program kinesiotherapeutic the functional autonomy of elderly. Methods: Interventional study with 20 elderly women, $70.5 \pm 6.7$ years old, good cognition and balance, the Reference Center for Social Assistance in Sao Luís- MA. Evaluation protocol was used functional autonomy of the Latin American Development Group for Maturity (GDLAM Protocol), before and after the intervention. The intervention consisted of kinesiotherapeutic program adapted from Montenegro and Silva (2007), with exercises that simulate AVDs, 60 minutes once a week for eight weeks. For data analysis, we used descriptive statistics. Results: There was reduction in the time required for participating doing all testing protocol, however, these rates were not enough to change the classification of the functional autonomy of the elderly, which remained weak. Regarding the test up from his chair and move around the house, older they evolved from regular to good. Conclusion: The results suggest that kinesiotherapeutic program may have contributed to that older diminish the time of achieving the GDLAM protocol testing and thereby improved functional autonomy of them. However, the scores were not enough to change the classification of the GDLAM standards in all tests. It is suggested to realization of longitudinal research, with more number of sessions of program.

Keywords: Aging; Functional Autonomy; Kinesiotherapy.

\section{Introdução}

Envelhecimento pode ser compreendido como um conjunto de alterações estruturais e funcionais desfavoráveis ao organismo que se acumulam de forma progressiva, especificamente em função do avanço da idade. Essas modificações prejudicam o desempenho de habilidades motoras, dificultando a adaptação do indivíduo ao meio ambiente e desencadeando alterações de ordem psicológica e social (CUNHA et al., 2009).

Atualmente, a autonomia ou capacidade funcional tem sido alvo de vários estudos, sobretudo em idosos, com o objetivo de avaliá-la, recuperá-la ou prevenir o seu declínio. Capacidade funcional se refere à potencialidade para desempenhar as atividades de vida diária (AVD's) ou de realizar determinado ato sem necessidade de ajuda, imprescindíveis para proporcionar uma melhor qualidade de vida (ALENCAR; BEZERRA; DANTAS, 2009; PEDROSA; HOLANDA, 2009).

Conforme Virtuoso Júnior e Guerra (2009), na velhice, as funções orgânicas decorrentes do processo de envelhecimento em consequência 
dos agravos ao longo da vida passam a ser mais vulneráveis ao declínio funcional, de forma que a capacidade funcional passa a ser um determinante da condição de saúde e bem-estar das pessoas.

Neste sentido, o Ministério da Saúde reconhece a preservação da capacidade funcional do idoso como um objetivo prioritário para a sua saúde. Na Política Nacional de Saúde da Pessoa Idosa (PNSPI), Portaria GM n ${ }^{\circ}$ 2.528, de 19 de outubro de 2006, a Capacidade Funcional é conceituada como a capacidade de manter habilidades físicas e mentais necessárias para uma vida independente e autônoma. Desta forma, trata-se de um enfoque que transcende o simples diagnóstico e tratamento de doenças específicas em idosos (BRASIL, 2006).

Segundo Alfieri, Teodori e Guirro (2006), o exercício físico regular tem sido reportado na literatura como fator benéfico à saúde por diminuir riscos potenciais de doenças, melhorar a capacidade cardiovascular e locomotora (aumento da densidade mineral óssea, melhora de sintomas ósteo-articulares, força muscular, equilíbrio, dentre outros), aumentando a capacidade funcional e promovendo melhores condições de vida aos idosos.

Varella e Jardim (2009) destacam que os músculos dos idosos são capazes de responder ao exercício de forma semelhante aos dos mais jovens, desde que treinados adequadamente. O exercício melhora a postura corporal e fortalece a musculatura ao redor das articulações, promovendo maior estabilidade e reduzindo as dores.

De acordo com César et al. (2004), mulheres idosas fisicamente ativas possuem maior independência funcional na realização de tarefas comuns do dia-a-dia quando comparadas a mulheres idosas sedentárias, sugerindo que a atividade física é uma ferramenta essencial na manutenção da autonomia e qualidade de vida das idosas.

Segundo Alencar, Henemann e Rothenbuhler (2008), a autonomia funcional surge como elemento fundamental para que o idoso possa ter uma vida independente, isto é, realizar suas atividades físicas e mentais necessárias para manutenção de suas atividades básicas como: tomar banho, vestir-se, alimentar-se, realizar higiene pessoal, arrumar a casa, fazer compras etc.

Dessa forma, Montenegro e Silva (2007) referem que a consciência dos efeitos que a idade exerce sobre a capacidade de aprender e executar as habilidades motoras coloca o fisioterapeuta em condições de ajudar o geronte, a aumentar sua participação numa série de atividades de lazer ou profissionais, além de orientá-lo a desenvolver sua capacidade de adaptação, em resposta às limitações físicas.

Considerando, então, que a capacidade funcional do idoso envolve uma gama de aspectos de sua vida e que o aumento da população idosa 


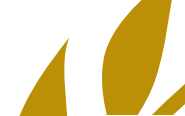

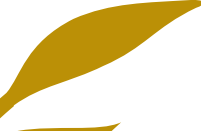

no mundo leva a necessidade de manter e melhorar a autonomia desses indivíduos, o presente estudo teve como objetivo analisar os efeitos de um programa de tratamento cinesioterapêutico na capacidade funcional de idosas.

\section{Materiais e Métodos}

Trata-se de um estudo descritivo, intervencionista realizado com 20 idosas, com média de idade de 70,5 \pm 67 anos, do Centro de Referência em Assistência Social (CRAS), no município de São Luís-MA.

Como critério de inclusão, foram considerados: idosos das instituições citadas, do gênero feminino, que concordassem em participar da pesquisa, com idade igual ou superior a 60 anos, que obtivessem equilíbrio e cognição satisfatórios comprovados, respectivamente, através da aplicação dos testes de Tinetti e Mini Mental. Foram excluídas idosas que apresentavam sequelas neurológicas, insuficiência cardíaca congestiva grave e quadros demenciais graves.

Assim, todas as possíveis participantes da pesquisa foram avaliadas inicialmente através dos testes de Equilíbrio de Tinetti, constituído de nove itens: equilíbrio sentado, levantando, tentativas de levantar, equilíbrio de pé imediato e em pé, desequilíbrio no esterno, teste dos três tempos: olhos fechados, girando $360^{\circ}$ e sentando, sendo que o escore aproximado ou igual a 16 corresponde ao grau satisfatório; e pelo Mini Mental, que fornece informações sobre parâmetros cognitivos contendo questões agrupadas em sete categorias: orientação temporal (5 pontos), orientação espacial (5 pontos), registro de três palavras (3 pontos), atenção e cálculo (5 pontos), recordação das três palavras (3 pontos), linguagem (8 pontos) e capacidade construtiva visual (1 ponto), sendo que o escore aproximado ou igual a 30 corresponde ao grau satisfatório. Todas as idosas avaliadas obtiveram êxito nos resultado dos testes.

No segundo momento, toda a amostra foi submetida aos testes do protocolo de avaliação da autonomia funcional do Grupo de Desenvolvimento Latino-Americano para a Maturidade (Protocolo GDLAM), constituídos por quatro testes: caminhar $10 \mathrm{~m}(\mathrm{C} 10 \mathrm{~m})$ levantar-se da posição sentada (LPS), levantar-se da posição decúbito ventral (LPDV) e levantar-se da cadeira e locomover-se pela casa (LCLC), aplicados nos locais da pesquisa, antes e após o protocolo de intervenção. Os resultados foram analisados na tabela padrão de avaliação da autonomia funcional do protocolo GDLAM, que classifica a autonomia funcional em fraco, regular, bom e muito bom (DANTAS; VALE; PERNAMBUCO, 2004).

Os equipamentos utilizados para os testes foram: trena, para precisar a distância; cronômetro, para marcar o tempo despendido na 
atividade; cadeira de braços com altura entre 43 e $50 \mathrm{~cm}$, dois cones e fita adesiva.

O protocolo utilizado consiste em um programa cinesioterapêutico adaptado de Montenegro e Silva (2007), que abrange exercícios que simulam as atividades de vida diária. Foi aplicado uma vez por semana, com sessões em grupo de 60 minutos, durante oito semanas.

O protocolo iniciava com dez minutos de alongamento de membros superiores e de membros inferiores. Seguia com vinte minutos de exercícios "ativo livre" e "ativo resistido" com garrafinhas pet preenchidas de areia para substituir halteres de $1 \mathrm{~kg}$ para os movimentos de flexão e extensão de cotovelos, seguidos de abdução e adução, flexão e extensão do ombro (simulando pegar objetos no alto).

Também foram inclusos os seguintes exercícios: com a idosa na posição sentada numa cadeira sem braços, realizar os movimentos de flexão e extensão de joelhos, flexão plantar e dorsiflexão dos tornozelos, trabalhando os gastrocnêmios e quadríceps (simulando apanhar objetos ou roupas no chão). Exercícios de propriocepção com balões e bastões sem carga, exercícios de coordenação para pescoço, mãos e pés (simulando dançar e limpar casa), associados a exercícios respiratórios (padrão diafragmático e padrão 3:1). Exercícios "ativo resistido" contra a gravidade de sentar e levantar da cadeira, partindo da posição sentada, exercícios "ativo resistido" na posição de pé, segurando atrás da cadeira, para membros inferiores alternando com flexão plantar dos tornozelos, flexão de quadril elevando os joelhos alternadamente, abdução do quadril alternadamente, trabalhando os flexores do quadril, abdutores e adutores, que colaboram nas tarefas de sentar e levantar de cadeiras, subir escadas e pegar ônibus.

O protocolo foi finalizado com vinte minutos de treinamento de marcha pela área da instituição, com a pesquisadora acompanhando e corrigindo a postura das idosas. Nos dez minutos finais, foram realizados alongamentos globais.

Todo o procedimento de aplicação dos exercícios foi realizado pela mesma pesquisadora.

Após as oito sessões da aplicação do protocolo de tratamento, foram realizadas as reavaliações da autonomia funcional, respeitando-se os mesmos procedimentos adotados durante a avaliação inicial.

A análise dos dados foi realizada, considerando a estatística descritiva.

\section{Resultados e discussão}

Os dados coletados foram utilizados para classificar a autonomia funcional das idosas participantes do estudo, de acordo com os padrões 
da GDLAM, em: fraco, regular, bom e muito bom (DANTAS; VALE; PERNAMBUCO, 2004). Esta classificação pode ser observada na Tabela 1.

Tabela 1 - Padrão de avaliação da autonomia funcional do protocolo GDLAM Testes

\section{Classificação C10M (seg.) LPS (seg.) LPDV (seg.) LCLC (seg.) IG (escore)}

\begin{tabular}{lccccc}
\hline Fraco & $>7,09$ & $>11,9$ & $>4,40$ & $>43,00$ & $>28,54$ \\
Regular & $7,08-6,34$ & $11,18-9,55$ & $4,39-3,30$ & $42,99-38,69$ & $28,53-25,25$ \\
Bom & $6,33-5,71$ & $9,54-7,89$ & $3,29-2,63$ & $38,67-34,78$ & $25,24-22,18$ \\
Muito bom & $<5,70$ & $<7,88$ & $<2,62$ & $<34,77$ & $<22,17$
\end{tabular}

Legenda: C10M = Caminhar 10 metros, LPS = Levantar da Posição Sentada, LPDV = Levantar da Posição de Decúbito Ventral, LCLC = Levantar da Cadeira e Locomover-se pela Casa. IG= índice GDLAM de autonomia funcional.

Fonte: Dantas; Vale e Pernambuco (2004).

Com base nesta classificação, na avaliação da autonomia funcional antes da intervenção, observou-se um valor considerado "fraco" de autonomia funcional para os testes C10m, LPS, LPDV e também para o índice GDLAM de autonomia funcional (IG). Na avaliação pós-intervenção, observou-se uma diminuição do tempo necessário para que as participantes realizassem todas as atividades propostas no protocolo, no entanto, estes índices não foram suficientes para alterar a classificação das idosas, que continuou sendo "fraco". Em relação ao teste LCLC, antes da intervenção, as idosas apresentavam um valor classificado como "regular" e, após a intervenção, foram classificadas como "bom" (Tabela 2).

Tabela 2-Avaliação da média do tempo, em segundo, dos testes de autonomia funcional com base no protocolo GDLAM, de idosas de São Luís, Maranhão.

\begin{tabular}{lccccc}
\hline $\begin{array}{c}\text { Testes do } \\
\text { GDLAM }\end{array}$ & \multicolumn{2}{c}{ Antes da Intervenção } & \multicolumn{3}{c}{ Depois da Intervenção } \\
\hline & $\begin{array}{c}\text { Valores em } \\
\text { segundos }\end{array}$ & Classificação & $\begin{array}{c}\text { Valores em } \\
\text { segundos }\end{array}$ & Classificação & $\Delta$ MA \\
\hline C10M & 11,8 & Fraco & 9,9 & Fraco & 1,9 \\
LPS & 15,65 & Fraco & 12,8 & Fraco & 2,85 \\
LPDV & 8,35 & Fraco & 6,15 & Fraco & 2,2 \\
LCLC & 41,45 & Regular & 36,85 & Bom & 4,6 \\
IG & 37,8 & Fraco & 31,55 & Fraco & 6,25 \\
\hline
\end{tabular}

Legenda: C10M = Caminhar 10 metros, LPS = Levantar da Posição Sentada, LPDV = Levantar da Posição de Decúbito Ventral, LCLC = Levantar da Cadeira e Locomover-se pela Casa. IG= índice GDLAM de autonomia funcional, $\triangle \mathrm{MA}=$ Diferença de Média Aritmética. 
Ao comparar os dados das tabelas 1 e 2, percebe-se que as participantes do estudo apresentavam, antes da aplicação do protocolo fisioterapêutico, valores muito acima dos escores considerados para classificação dos testes como "fraco", expostos na tabela 1, indicando que as idosas pesquisadas apresentavam restrita autonomia funcional. Isto pode estar relacionado ao fato de que as idosas estudadas não participavam de atividades físicas regulares (SANTOS et al., 2010).

Estes resultados são semelhantes aos de outros estudos (ARAGÃO; DANTAS; DANTA, 2003; CÉSAR et al.,2004; Perez et al., 2010), em que a média dos grupos estudados foi considerada como fraco em todos os itens. No estudo de César et al. (2004),a única exceção foi o teste LCLC, que obteve a classificação "muito bom".

Por outro lado, na presente pesquisa, observou-se diferenças entre as médias aritméticas em relação aos escores de todos os testes ao comparar os valores antes e após a aplicação do protocolo cinesioterapêutico, sugerindo que este protocolo produziu efeitos benéficos para tais atividades.

Os testes realizados neste estudo mediram a capacidade de locomoção e a independência da amostra na realização de atividades cotidianas. Salienta-se que a capacidade de realização das atividades da vida diária garante ao idoso maior autonomia no seu dia-a-dia (VALE; DANTAS, 2008).

Utiliza-se o índice GDLAM para associar as atividades de vida diária e a autonomia funcional, representando o nível desta variável nos indivíduos idosos. O índice verifica o nível de independência funcional que o idoso se encontra, através dos resultados obtidos em segundos para a realização dos quatro testes (C10M, LPS, LPDV e LCLC) que reproduzem situações com as quais o idoso se depara no dia-a-dia, como subir uma escada, sentar-se ou levantar-se de uma cadeira, entrar em um ônibus e atravessar uma rua e o programa de cinesioterapia foi realizado com exercícios que simulavam essas situações cotidianas, como: levantar-se do chão ou de uma cama, desviar-se de obstáculos no caminho, pegar objetos no chão e varrer casa, por exemplo (DANTAS; VALE; PERNAMBUCO, 2004).

O propósito dos testes C10M e LCLC é, respectivamente, avaliar a velocidade que o indivíduo leva para percorrer a distância de 10 metros e avaliar a capacidade do idoso na sua agilidade e equilíbrio em diversas situações de sua vida (GURALNIK et al., 2000).

O propósito dos testes LPDV e LPS, respectivamente, é avaliar a habilidade do indivíduo para levantar-se do chão e avaliar a capacidade funcional da extremidade inferior. Atividades que dispõem da rápida movimentação no espaço ou mudança repentina de direção da extremidade inferior podem ser realizadas com mais dificuldade com o avanço da idade. 


\section{de

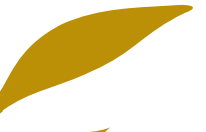

Segundo Matsudo, Matsudo e Barros Neto (2000), idosos fisicamente ativos apresentam um maior grau de autonomia em atividades que requerem força de membros inferiores e independente do mecanismo envolvido, a melhora da força muscular tem relação direta com a melhora na capacidade de subir escada e se levantar de uma posição sentada.

No teste LCLC, o escore de autonomia funcional das idosas evoluiu de regular para bom. Destaca-se que esse teste associa as habilidades necessárias para os testes levantar-se da posição sentada (LPS) e caminhar 10 metros (C10M), sugerindo que o protocolo também influenciou positivamente nestas habilidades, mesmo que não tenha havido alteração no nível de autonomia para tais testes.

Uma vez que a idade mostra-se um fator fortemente associado à perda da autonomia funcional, torna-se importante manter a aptidão física em indivíduos idosos, pois quanto maior a idade, maior o tempo para a realização de tarefas. Para Hurley e Hoth (2000), a melhor opção para o indivíduo que está envelhecendo é a realização de um programa de atividade física que incorpore exercícios específicos.

Os estudos de Cesar et al. (2004) indicaram que idosos fisicamente ativos possuem uma ordem de locomoção e deslocamento mais eficientes do que idosos sedentários, o que Ihes provê maior autonomia cotidiana em atividades como atravessar uma rua ou se locomover sem ajuda de terceiros.

Logo, para Guimarães et al. (2004), a manutenção e a preservação da capacidade para desempenhar as AVD's são pontos básicos para prolongar a independência do idoso. A reabilitação gerontológica visa a preservação da função, adiamento da instalação de incapacidades, tendo como objetivo diminuir o comprometimento imposto por elas, promovendo um modo de vida mais saudável e adaptando o indivíduo de forma a propiciar uma melhor qualidade de vida.

Evidencia-se que esta pesquisa se restringiu a analisar variáveis relacionadas ao desempenho motor, no entanto, sabe-se que a prática de exercícios podem apresentar outros benefícios como: melhora da autoestima, maior envolvimento social e menores riscos de doenças associadas à hipocinesia (RIGO; TEIXEIRA, 2005). 


\section{Conclusão}

Os resultados obtidos neste estudo sugerem que o programa cinesioterapêutico utilizado foi capaz de diminuir o tempo de realização de atividades de vida diária das idosas pesquisadas e, por conseguinte, melhorar a autonomia funcional delas. No entanto, os escores obtidos após a intervenção não foram suficientes para alterar a classificação das idosas quanto aos padrões da GDLAM.

Destaca-se que o número restrito de sessões do programa ciensioterapêutico utilizado pode ter influenciado este resultado. Diante disso, sugerem-se novas pesquisas de caráter longitudinal, que possam aplicar este programa com um número maior de sessões.

Considera-se que programas de orientações e incentivo sobre os benefícios da atividade física são recomendados para que os idosos mantenham sua capacidade funcional $e$, consequentemente, tenham melhor qualidade de vida, pois idosas que permanecem inativas fisicamente ao longo da vida sofrerão os efeitos do envelhecimento com maior impacto.

\section{REFERÊNCIAS}

ALENCAR, M. C. B.; HENEMANN, L.; ROTHENBUHLER, R. A Capacidade funcional de pacientes, e a fisioterapia em um programa de assistência domiciliar. Fisioterapia em Movimento, Paraná, v. 21, n. 1, p. 11-20, jan./mar.2008.

ALENCAR, N. A.; BEZERRA, J. C.; DANTAS, E. H. M. Avaliação dos níveis de atividade física, autonomia funcional e qualidade de vida de idosas integrantes da estratégia saúde da família. Fitness \& Performance Journal, Rio de Janeiro, v. 8, n. 5, p. 21-315, set./out. 2009.

ALFIERI, F. M.; TEODORI, R. M.; GUIRRO, R. R. J. Estudo Baropodométrico em idosos submetidos à intervenção fisioterapêutica. Fisioterapia em Movimento, Curitiba, v. 19, n. 2, p. 67-74, abr./jun. 2006.

ARAGÃO, J. C.; DANTAS, E. H.; DANTAS, B. H. Efeitos da resistência muscular localizada visando a autonomia funcional e a qualidade de vida do idoso. Fitness \& Performance Journal, v.1, n.3, p. 29-37, 2003.

BRASIL Ministério da Saúde. Política Nacional de Saúde da Pessoa Idosa, Lei 8,842/94, Portaria 2.528 de 19 de outubro de 2006, p2024, Brasília DF. 
CESAR, E. P. et al. Aplicação de quatro testes do protocolo GDLAMgrupo de desenvolvimento latino-americano para maturidade. Revista Mineira de Educação Física, Viçosa, v. 12, n. 1, p. 18-37, 2004.

CUNHA, M. F. et al. A influência da fisioterapia na prevenção de quedas em idosos na comunidade: estudo comparativo. Motriz Revista de Educação Física, Rio Claro, v. 15, n. 3, p. 527-536, 2009.

DANTAS, E. H. M.; VALE, R. G. S.; PERNAMBUCO, C. S. Protocolo GDLAM na Avaliação da Autonomia Funcional. Fitness \& Performance Journal, Rio de Janeiro, v. 3, n. 3, p. 175-183, maio/jun. 2004.

GURALNIK, J. M. et al. Lower extremity function and subsequent disability: consistency across studies, predictive models, and value of gait speed alone compared with the short physical performance battery, Journals of Gerontology, Series A: Biological Sciences and Medical Sciences, n. 55, p.221-231.

GUIMARAES, L. H. T. C. et al. Avaliação da capacidade funcional de idosos em tratamento fisioterapêutico. Revista Neurociências, v. 12 n. 3, jul./set. 2004.

HURLEY, B. F.; ROTH, S. M. Strength training in the elderly: effects on risk factors for age-related diseases. Sports Medicine, v. 30, n. 4, p. 249-268, 2000.

MATSUDO, S. M.; MATSUDO, V. K. R.; BARROS NETO, T. L. Impacto do envelhecimento nas variáveis antropométricas, neuromotoras e metabólicas da aptidão física. Revista Brasileira de Ciência e Movimento, v. 8, n. 4 , p. $21-32,2000$.

MONTENEGRO, S. M. R. S.; SILVA, C. A. B. Os efeitos de um programa de fisioterapia como promotor de saúde na capacidade funcional de mulheres idosas institucionalizadas. Revista Brasileira de Geriatria e Gerontologia, Rio de Janeiro, v. 10, n. 2, 2007.

PEDROSA, R.; HOLANDA, G. Correlação entre os testes da caminhada, marcha estacionária e tug em hipertensas idosas. Revista Brasileira de Fisioterapia, São Carlos, v. 13, n. 3, p. 252-6, maio/jun. 2009. 
PEREZ, A. J. et al. Estudo comparativo da autonomia de ação de idosas praticantes e não praticantes de exercícios físicos regulares. Rev Bras Med Esporte, v.16, no.4, p.254-258, Ago. 2010.

RIGO, M. L. N. R.; TEIXEIRA, D. C. Efeitos da atividade física na percepção de bem-estar de idosas que residem sozinhas e acompanhadas. Revista Unopar Científica Ciências Biológicas e da Saúde, v. 7, n. 1, p. 1320, 2005.

SANTOS, L. T. A. et al . Efeitos da cinesioterapia sobre os níveis de IGF-1, força muscular e autonomia funcional em mulheres idosas. Rev. bras. cineantropom. desempenho hum., Florianópolis, v. 12, n. 6, p. 451-456, dez. 2010.

VALE, R. G. S; DANTAS, E. H. M. Autonomia funcional do idoso. In: Dantas, E. H. M, VALE, R. G. S. Atividade física e envelhecimento saudável. Rio de Janeiro: Shape,2008.

VARELLA, D.; JARDIM, C. Guia prático de saúde e bem-estarenvelhecimento. São Paulo: Gold Editora, 2009. Coleção Doutor Drauzio Varella.

VIRTUOSO JÚNIOR, J. S.; GUERRA, R. O. Incapacidade funcional em mulheres idosas de baixa renda. Ciência \& Saúde Coletiva, v. 16, n. 5, p. 2541-2548, 2011. 